\title{
Piezoelectric Accelerometer Modification Based on the Finite Element Method
}

\author{
B. Liu ${ }^{\dagger}$ and B. Kriegbaum \\ BrLel \& Kj6r Sound \& Vibration Measurement A/S, Skodsborgvej307, 2850 N6rum, Denmark
}

(Received 15 September 1999; accepted 12 January 2000)

\begin{abstract}
The paper describes the modification of piezoelectric accelerometers using a Finite Element (FE) method. B[ el \& K $] \quad$ r Accelerometer Type 8325 is chosen as an example to illustrate the advanced accelerometer development procedure. The deviation between the measured and FE simulated results of Accelerometer Type 8325 is below $6 \%$. It is proved that the specifications of the accelerometer can be effectively predicted using the FE method, especially when modifications of the accelerometer are required.
\end{abstract}

${ }^{\dagger}$ Member of the International Institute of Acoustics and Vibration (IIAV)

\section{INTRODUCTION}

Piezoelectric accelerometers are widely used for vibration measurements. They were first commercialised in the 1940s. ${ }^{1}$ Generally piezoelectric accelerometers have been modelled using analytical and combined analytical-empirical methods. The analytical method is simplified to an equivalent circuit method that is developed on the basis of the electromechanical analogy. As a one-dimensional method, the equivalent circuit model has limited accuracy and cannot be used for two- or three-dimensional analysis because it normally ignores the coupling effects between different modes.

With the advent of inexpensive and fast computers, numerical techniques have been developed rapidly which now can solve present-day physical problems in engineering analysis and design. The FE method is one such technique. It can be used flexibly to model any arbitrary geometry and characterise any given property of the material.

For piezoelectric accelerometer modelling, the basic FE method is used purely for mechanical analysis, e.g. to determine resonance modes, stresses, and other parameters. In addition to determining these properties, the most challenging task is to simulate the electrical characteristics of the piezoelectric accelerometers and optimise the electric sensitivities as a function of frequency, while keeping the resonance frequency as high as possible using the FE method.

In the numerical work presented in this paper, the ANSYS $®$ code has been used.

\section{THE FE METHOD FOR ACCELEROMETERS}

In the FE simulation of accelerometers, the first step is to divide the accelerometer structure into a finite number of small regions, i.e. the FE meshes. Each element is specified by a discrete number of points, namely, the nodes of the element. The positions of the nodes are arranged in the global co-ordinate system. Within each element the variables are given by some interpolation rules in terms of their values at the nodes. Their values at any point within the element are obtained by interpolation between the discrete values at the nodes of the element in conjunction with the properties on the boundaries represented by boundary condition and loads. Different mechanical and vibration conditions are also simulated and loaded on the FE model while the desired performances are calculated.

In this paper, the B $[\quad$ el \& K $] \quad \mathrm{r}$ Accelerometer Type 8325 is chosen as an example to explain how the modification of piezoelectric accelerometers is made using the FE method. Type 8325 is an Annular Shear type accelerometer with an insulated and double-shielded case. ${ }^{2}$

Figure 1 shows the mesh of the accelerometer generated by the ANSYS ${ }^{\circledR}$ program. The structure and ceramic material were modelled with element SOLID45 and element SOLID5 defined in ANSYS $\AA$, respectively. ${ }^{3}$ The piezoelectric material was modelled using the material parameters for PZ23. ${ }^{4}$ These parameters are shown in Table 1. The PZ material was poled in the upward direction in the thickness dimension. The structure was modelled as 3-D solid material. Table 2 shows the properties used in the model for these materials.

The measurement and FE simulation charge sensitivity plots of Type 8325 are shown in Fig. 2. The slopes in the charge sensitivity plots are due to the frequency-dependent piezoelectric constant $d_{15}$. The slope should have a value of $2.5 \%$ per decade. The slope might not be regular, especially in the low frequency regime. This could be the explanation for the deviation between the measurement and FE simulation results shown in Fig. 2. In the high frequency regime, the deviation becomes larger. This is due to the fact that mechanical and electrical losses had not been included in the FE simulation. Furthermore, the losses are significant around the resonance frequency.

A comparison of the most important specifications between the measurement and FE simulation results is shown in Table 3 .

The charge sensitivity is calculated at the frequency of 159.2 Hz, using ANSYS® Harmonic Analysis, by applying acceleration on the mounted surface of the FE model. The mounted resonance frequency was calculated by using ANSYS ${ }^{\circledR}$ Modal Analysis. The mounted resonance frequency is the frequency at which the accelerometer operates up and down. The deformation shape is shown in Fig. 3, which only includes the piezoelectric material and the mass in order to make the picture readable. 